\title{
A New England COVID-19 Registry of Patients With CNS Demyelinating Disease
}

\section{A Pilot Analysis}

Kelli M. Money, MD, PhD, Ashmanie Mahatoo, MD, Soleil Samaan, Pria Anand, MD, Ursela Baber, MD, Mary Bailey, MD, Rohit Bakshi, MD, Andrew Bouley, MD, Aaron Bower, MD, Jonathan Cahill, MD, Maria Houtchens, MD, Joshua Katz, MD, Ellen Lathi, MD, Elle Levit, MD, Erin E. Longbrake, MD, PhD, Matthew McAdams, MD, Salvatore Napoli, MD, Pooja Raibagkar, MD, Peter Wade, MD, and Jacob A. Sloane, MD, PhD

Neurol Neuroimmunol Neuroinflamm 2021;8:e1046. doi:10.1212/NXI.0000000000001046

\section{Abstract}

\section{Background and Objectives}

We sought to define the risk of severe coronavirus disease 2019 (COVID-19) infection requiring hospitalization in patients with CNS demyelinating diseases such as MS and the factors that increase the risk for severe infection to guide decisions regarding patient care during the COVID-19 pandemic.

\section{Methods}

A pilot cohort of 91 patients with confirmed or suspected COVID-19 infection from the Northeastern United States was analyzed to characterize patient risk factors and factors associated with an increased severity of COVID-19 infection. Univariate analysis of variance was performed using the Mann-Whitney $U$ test or analysis of variance for continuous variables and the $\chi^{2}$ or Fisher exact test for nominal variables. Univariate and stepwise multivariate logistic regression identified clinical characteristics or symptoms associated with hospitalization.

\section{Results}

Our cohort demonstrated a $27.5 \%$ hospitalization rate and a $4.4 \%$ case fatality rate. Performance on Timed 25-Foot Walk before COVID-19 infection, age, number of comorbidities, and presenting symptoms of nausea/vomiting and neurologic symptoms (e.g., paresthesia or weakness) were independent risk factors for hospitalization, whereas headache predicted a milder course without hospitalization. An absolute lymphocyte count was lower in hospitalized patients during COVID-19 infection. Use of disease-modifying therapy did not increase the risk of hospitalization but was associated with an increased need for respiratory support.

\section{Discussion}

The case fatality and hospitalization rates in our cohort were similar to those found in MS and general population COVID-19 cohorts within the region. Hospitalization was associated with increased disability, age, and comorbidities but not disease-modifying therapy use.

\author{
Correspondence \\ Dr. Sloane \\ jsloane@bidmc.harvard.edu
}

From the Department of Neurology (K.M.M., A.M., S.S., U.B., J.A.S.), Beth Israel Deaconess Medical Center, Boston; Department of Neurology (P.A.), Boston University School of Medicine, MA; The Mandell Comprehensive MS Center (M.B., P.W.), Hartford, CT; Department of Neurology (R.B., M.H.), Brigham and Women's Hospital, Boston; The Elliot Lewis Center (A. Bouley, J.K., E. Lathi), Wellesley, MA; Department of Neurology (A. Bower, E. Levit, E.E.L.), Yale School of Medicine, New Haven, CT; Department of Neurology (J.C.), Alpert Medical School of Brown University, Providence, RI; Department of Neurology (M.M.), Lahey Hospital, Burlington; Neurology Center of New England (S.N.), Foxboro, MA; and Department of Neurology (P.R.), Concord Hospital, Concord, NH.

Go to Neurology.org/NN for full disclosures. Funding information is provided at the end of the article. 


\section{Glossary}

25FTW = Timed 25-Foot Walk; ALC = absolute lymphocyte count; ANOVA = analysis of variance; BMI = body mass index; COVID-19 = coronavirus disease 2019; DMT = disease-modifying therapy; EDSS = Expanded Disability Status Scale; ICU = intensive care unit.

In the past year, coronavirus disease 2019 (COVID-19) has become the third worst pandemic in modern history, with almost 100 million cases and over 2 million deaths worldwide as of January $2021 .^{1}$ It has ravaged the most vulnerable populations with high mortality rates among the elderly, those with a disability, and those with medical comorbidities. ${ }^{2,3}$

Neurologists were initially concerned that patients with MS and other CNS demyelinating diseases would have more severe adverse effects of COVID-19 and have worked quickly to identify COVID-19 risks in the MS population. Prior work indicates that patients with MS, particularly those with substantial disability, are at an increased risk of infectious illnesses. ${ }^{4}$ The various forms of immunomodulatory treatments may also increase the risk of infection and subsequent hospitalization and death. ${ }^{5}$ In addition, there has been a concern that COVID-19 may increase the risk of MS relapse or worsen preexisting MS symptoms (i.e., pseudorelapse). Many patients have chosen to alter their disease-modifying therapy (DMT) dosing schedule without input from their neurologists, ${ }^{6}$ and professional guidance on altered DMT dosing or the initiation schedule has been based on limited to no evidence. $^{7,8}$

Here, we present a pilot analysis of a cohort of 91 patients (86 MS and 5 non-MS CNS demyelinating diseases) with confirmed or suspected COVID-19 from the MS centers within the New England states in the northeastern United States. We hypothesized that COVID-19 severity would be associated with disability but not treatment given prior reports of mildmoderate COVID-19 infection while on DMTs. ${ }^{9-12}$ We also sought to identify predictive factors related to COVID-19 severity in this patient population, including laboratory tests.

\section{Methods}

We collected physician-reported data through the survey from 8 New England institutions (eAppendix, links.lww.com/NXI/ A513). Patients were included if they had PCR or antibodyconfirmed COVID-19 or if COVID-19 was suspected based on typical symptoms. ${ }^{13}$ Patients with MS, neuromyelitis optica spectrum disorder, clinically isolated syndrome, radiologically isolated syndrome, and other neuroimmunologic disorders were included. We used an electronic record form on REDCap (project-redcap.org/) to collect study data for all sites. Data included were collected before November 2020, but follow-up data from the current cohort and additional cases continue to be collected through the survey from participating MS centers. A few patients have been prior published by the contributing physicians as case reports but have not been included in other cohorts.

Analyses were conducted using Microsoft Excel 2013 (Redmond, WA) for descriptive statistics and graphing and JMP Pro 15 statistical software (Cary, NC) for more complex statistics such as univariate and multivariate regressions. Laboratory values, age, body mass index (BMI), number of non-neuroimmunologic comorbidities, neuroimmunologic disease duration, Expanded Disability Status Scale (EDSS), Timed 25-Foot Walk (25FTW), COVID-19 symptom duration, and COVID-19 PCR positivity duration were treated as continuous variables, with the remaining variables as nominal. BMI greater than 30 was considered obese. MS relapse or pseudorelapse was determined by physician-submitting case data, as imaging data were not available for analysis. Hospitalization status, intensive care unit (ICU) admission, and need for respiratory support were reported, although indication for escalation of care was not specified in the data collection. Headache was separated from neurologic symptoms given the frequency of association with COVID-19 infection. DMTs were analyzed as individual treatments, grouped as any DMT vs no DMT, grouped based on efficacy, and grouped based on the mechanism of action. Rituximab, ocrelizumab, and natalizumab were categorized as highefficacy treatments; dimethyl fumarate, fingolimod, and cladribine as mid-efficacy treatments; and teriflunomide, glatiramer acetate, and interferon-B as low-efficacy treatments. Multiple imputations with singular value decomposition were used for missing continuous variables, specifically BMI, EDSS, and 25FTW. This method was not used for laboratory values because of limited data availability. Unknowns in nominal categories were presumed as an absence of symptom/ condition or folded into the lower severity category as indicated in Tables 1 and 2 . The number of missing data points for each continuous variable can be found in the Figure/Table or the associated legend. Continuous variables with missing data points that were not able to be addressed with multiple imputations were not included in the regression analysis. Univariate analysis was with the Mann-Whitney $U$ test or analysis of variance (ANOVA) for continuous variables and the $\chi^{2}$ or Fisher exact test for nominal variables. Univariate nominal logistic regression was run with a target value of "yes." Multivariate nominal logistic regression was run in a stepwise fashion based on Akaike information criteria and mixed-effects modeling. Laboratory values were analyzed through two-way ANOVA with significance between individual groups determined by Bonferroni post hoc analysis. Significance was considered at $p<0.05$ in all analyses. 
Table 1 Case Demographics

All cases $(n=91)$

Gender, \% (n)

Male

Female

Nonbinary

Age, average (SEM)

Race, \% (n)

\begin{tabular}{l} 
White \\
\hline Black \\
\hline Other/UNK
\end{tabular}

$65.9(60)$

$18.7(17)$

$15.4(14)$
Nonhospitalized $(n=66)$

Hospitalized $(n=25)$

Ethnicity, \% (n)

\begin{tabular}{|c|c|c|c|}
\hline Hispanic & $14.3(13)$ & $13.6(9)$ & $16.0(4)$ \\
\hline BMI, average (SEM) & $30.8(0.9)$ & $30.9(1.1)$ & $30.4(1.6)$ \\
\hline \multicolumn{4}{|l|}{ Tobacco use, $\%$ (n) } \\
\hline Current & $15.4(14)$ & $18.2(12)$ & $8.0(2)$ \\
\hline Prior & $27.5(25)$ & $25.8(17)$ & $32.0(8)$ \\
\hline Never/UNK & $57.1(52)$ & $56.1(37)$ & $60.0(15)$ \\
\hline \multicolumn{4}{|l|}{ Employment, \% (n) } \\
\hline Working & $50.5(46)$ & $57.6(38)$ & $32.0(8)$ \\
\hline Disability & $27.5(25)$ & $21.2(14)$ & $44.0(11)$ \\
\hline Retired/unemployed/UNK & $22(20)$ & $21.2(14)$ & $24.0(6)$ \\
\hline No. of comorbidities, average (SEM) & $2.4(0.2)$ & $1.9(0.2)^{\mathrm{a}}$ & $3.7(0.5)^{a}$ \\
\hline Any comorbidity, \% (n) & $75.8(69)$ & $72.7(48)$ & $84.0(21)$ \\
\hline Obesity, \% (n) & $30.8(28)$ & $31.2(21)$ & $28.0(7)$ \\
\hline Cardiovascular disease, \% (n) & $7.7(7)$ & $4.5(3)^{a}$ & $16.0(4)^{a}$ \\
\hline Hypertension, \% (n) & $34.1(31)$ & $25.8(17)^{a}$ & $56.0(14)^{a}$ \\
\hline Hyperlipidemia, \% (n) & $25.3(23)$ & $21.2(14)$ & $36.0(9)$ \\
\hline Diabetes, \% (n) & $12.1(12)$ & $12.1(8)$ & $12.0(3)$ \\
\hline Cancer, \% (n) & $4.4(4)$ & $0(0)^{a}$ & $16.0(4)^{a}$ \\
\hline Chronic lung disease, $\%(n)$ & $12.1(11)$ & $10.6(7)$ & $16.0(4)$ \\
\hline \multicolumn{4}{|l|}{ MS type, \% (n) } \\
\hline RRMS & $71.4(65)$ & $78.8(52)^{a}$ & $52.0(13)^{a}$ \\
\hline SPMS/PPMS & $23.1(21)$ & $16.7(11)^{a}$ & $40.0(10)^{a}$ \\
\hline Other (NMO/unspecified) & $5.5(5)$ & $4.5(3)^{a}$ & $8.0(2)^{a}$ \\
\hline Disease duration, average years (SEM) & $15.7(1.2)$ & $14.5(1.3)$ & $19.0(2.3)$ \\
\hline EDSS, average (SEM) & $4.0(0.3)$ & $3.6(0.3)^{a}$ & $5.0(0.5)^{a}$ \\
\hline 25FTW, average (SEM) & $6.6(0.3)$ & $6.2(0.2)^{a}$ & $7.9(0.7)^{a}$ \\
\hline Steroids within 1 mo, \% (n) & $5.5(5)$ & $3.0(2)$ & $12.0(3)$ \\
\hline
\end{tabular}

Current DMT (n)
$15.2(10)$

$12.0(3)$

$32.0(8)$

$68.0(17)$

$0(0)$

$58.8(2.3)^{a}$

$47.8(1.7)^{a}$

72.0 (18)

12.0 (3)

$0(2)$

25.8 (17)

$44.0(11)$

$24.0(6)$

$3.7(0.5)^{a}$

84.0 (21)

$28.0(7)$

$6.0(4)^{a}$

$56.0(14)^{a}$

$16.0(4)$

$52.0(13)^{\mathrm{a}}$

$40.0(10)^{a}$

$5.5(5)$

$15.7(1.2)$

$.0(0.3)$

5.5 (5)

$3.0(2)$

$12.0(3)$ 
Table 1 Case Demographics (continued)

\begin{tabular}{llll}
\hline & All cases $(\mathbf{n}=\mathbf{9 1})$ & Nonhospitalized $(\mathbf{n}=\mathbf{6 6})$ & Hospitalized $(\mathbf{n}=\mathbf{2 5})$ \\
\hline None & 22 & 15 & 7 \\
\hline Rituximab/ocrelizumab & 30 & 20 & 10 \\
\hline Dimethyl fumarate & 7 & 6 & 1 \\
\hline Glatiramer acetate & 6 & 5 & 1 \\
\hline Natalizumab & 13 & 12 & 1 \\
\hline Interferon & 4 & 3 & 1 \\
\hline Cladribine & 1 & 0 & 1 \\
\hline Fingolimod & 3 & 3 & 0 \\
\hline Teriflunomide & 2 & 2 & 0 \\
\hline Other/UNK & 3 & 0 & 3
\end{tabular}

Abbreviations: 25FTW = Timed 25-Foot Walk; BMI = body mass index; COVID-19 = coronavirus disease 2019; DMT = disease-modifying therapy; EDSS = Expanded Disability Status Scale; NMO = neuromyelitis optica; PPMS = primary progressive MS; RRMS = relapsing-remitting MS; SPMS = secondary progressive MS; UNK = unknown.

Clinical characteristics for each COVID-19 case are listed. All cases include all patients in the data set (second from the left column). These cases were then separated by hospitalization status (nonhospitalized [second from the right column] and hospitalized [right column]). There were significant differences between nonhospitalized and hospitalized patients by the $\chi^{2}$ or Mann-Whitney $U$ test with regard to age, number of comorbidities, cardiovascular disease, hypertension, hyperlipidemia, cancer, MS type, EDSS, and 25FTW. Values are expressed as either percentages with $\mathrm{n}$ in parenthesis or averages with SEM in parenthesis. Missing continuous variable data points were addressed by multiple imputations. Before analysis, the missing data points for nonhospitalized included 15 for BMI, 18 for EDSS, and 24 for 25FTW; and for hospitalized patients included 4 for BMI, 9 for EDSS, and 14 for 25FTW.

a Significance of $p<0.05$.

\section{Standard Protocol Approvals, Registrations, and Patient Consents}

The study received approval from the Beth Israel Deaconess Medical Center Institutional Review Board committee (Protocol\# 2020P000343), and institutional review board approval was ceded for other institutions involved in this study.

\section{Data Availability}

Raw data can be requested from the corresponding author(s), and we will continue to accept reports of additional COVID-19 cases among patients with CNS demyelinating diseases to include in our New England CNS demyelinating disease COVID-19 registry by contacting the corresponding author(s).

\section{Results}

Our cohort consists of 91 patients with CNS demyelinating disease with confirmed or suspected COVID-19 from provider reports within the New England region. The clinical characteristics, COVID-19 symptoms, and outcome measures are included in Tables 1 and 2. Most patients were female (69.2\%), White (65.9\%), and non-Hispanic (85.7\%). $75.8 \%$ of patients had at least 1 medical comorbidity with obesity $(30.8 \%)$ and hypertension (34.1\%) being the most common. Most patients had relapsing/remitting MS (71.4\%) and were on a DMT (75.8\%). The average neuroimmunologic disease duration was 15.7 years, and the average EDSS was 4.0.

Outcome measures were $11.0 \%$ asymptomatic, $27.5 \%$ hospitalized, $13.2 \%$ requiring respiratory support, $5.5 \%$ admitted to the ICU, and $4.4 \%$ mortality. Of the symptoms reported at the time of COVID-19 diagnosis, fever (48.4\%), cough (41.8\%), and fatigue $(34.1 \%)$ were most frequently observed. There were several patients with concomitant non-COVID-19 infections. Three patients had urinary tract infections; 1 patient had methicillin-susceptible Staphylococcus aureus bacteremia; 1 patient had Clostridium difficile; and 1 patient had an unspecified bacterial coinfection. It is unknown whether any of the unspecified pathogens were opportunistic infections. There were 2 MS relapses and 5 pseudorelapses reported during COVID-19 infection or within 1 month of recovery. None were on a high-efficacy DMT ( 3 were on no DMT, 3 on low-efficacy DMTs, and 1 on a mid-efficacy DMT). Two patients received high-dose steroids immediately before diagnosis: one on glatiramer acetate requiring hospitalization with full recovery and the other on ocrelizumab not requiring hospitalization.

\section{Factors Associated With Hospitalization}

Several unique characteristics were appreciated when comparing hospitalized patients with nonhospitalized patients as demonstrated in Tables 1 and 2. Hospitalized patients were older (average age 58.8 vs 47.8 years, $p<0.001$ ) and had more medical comorbidities (3.7 vs $1.9, p<0.01)$ such as cardiovascular disease $(16.0 \%$ vs $4.6 \%, p<0.05)$, hypertension ( $56.0 \%$ vs $25.8 \%, p<0.05)$, and cancer $(16.0 \%$ vs $0.0 \%, p<$ 0.01 ). Numbers per cancer type (lung, colon, leukemia, and unspecified) were too small to assess statistically. Patients with MS showing a progressive phenotype $(40.0 \%$ vs $16.7 \%, p<0.05)$ with higher disability scores (EDSS 5.0 vs $3.6, p<0.05$; $25 \mathrm{FTW}$ 
Table 2 Symptoms and Outcome Metrics for All Cases

\begin{tabular}{|c|c|c|c|}
\hline & All cases $(n=91)$ & Nonhospitalized $(n=66)$ & Hospitalized $(n=25)$ \\
\hline Confirmed diagnosis, \% (n) & $74.7(68)$ & $74.2(49)$ & $76.0(19)$ \\
\hline Symptomatic, \% (n) & $89(81)$ & $84.8(56)$ & $100.0(25)$ \\
\hline Symptom duration, average days (SEM) & $12.8(1.9)$ & $12.9(1.9)$ & $12.7(6.7)$ \\
\hline Diagnosis duration, average days (SEM) & $39.9(5.3)$ & $41.3(6.0)$ & $36.8(11.4)$ \\
\hline \multicolumn{4}{|l|}{ Presenting symptoms, \% (n) } \\
\hline Fever & $48.4(44)$ & $45.5(30)$ & $56.0(14)$ \\
\hline Cough & $41.8(38)$ & $37.9(25)$ & $48.0(12)$ \\
\hline Rhinorrhea & $8.8(8)$ & $9.1(6)$ & $8.0(2)$ \\
\hline Fatigue & $34.1(31)$ & $30.3(20)$ & $40.0(10)$ \\
\hline Pain & $20.9(19)$ & $21.2(14)$ & $20.0(5)$ \\
\hline Myalgia & $24.2(22)$ & $24.2(16)$ & $24.0(6)$ \\
\hline Chest pain & $9.9(9)$ & $7.6(5)$ & $16.0(4)$ \\
\hline Sore throat & $17.6(16)$ & $18.2(12)$ & $16.0(4)$ \\
\hline Dyspnea & $30.8(28)$ & $24.2(16)^{a}$ & $44.0(11)^{a}$ \\
\hline Chills & $19.8(18)$ & $21.2(14)$ & $16.0(4)$ \\
\hline Dysgeusia & $23.1(21)$ & $24.2(16)$ & $20.0(5)$ \\
\hline Anosmia & $20.9(19)$ & $22.7(15)$ & $16.0(4)$ \\
\hline Headache & $27.5(25)$ & $34.8(23)^{a}$ & $8.0(2)^{a}$ \\
\hline Neurologic & $25.3(23)$ & $13.6(9)^{a}$ & $56.0(14)^{a}$ \\
\hline Diarrhea & $15.4(14)$ & $12.1(8)$ & $24.0(6)$ \\
\hline Nausea/vomiting & $14.3(13)$ & $7.6(5)^{\mathrm{a}}$ & $32.0(8)^{a}$ \\
\hline Loss of appetite & $9.9(9)$ & $9.1(6)$ & $12.0(0)$ \\
\hline Pneumonia, \% (n) & $11(10)$ & $0(0)$ & $40.0(10)$ \\
\hline Hospitalized, \% (n) & $27.5(25)$ & $\mathrm{N} / \mathrm{A}$ & $\mathrm{N} / \mathrm{A}$ \\
\hline Intubated or admitted to the ICU, \% (n) & $5.5(5)$ & $\mathrm{N} / \mathrm{A}$ & $20.0(5)$ \\
\hline \multicolumn{4}{|l|}{ Outcome, \% (n) } \\
\hline Asymptomatic & $11.0(10)$ & $15.2(10)$ & $0(0)$ \\
\hline Recovered/recovering/UNK & $84.6(77)$ & $83.3(55)$ & $88.0(25)$ \\
\hline Death & $4.4(4)$ & $1.5(1)$ & $12.0(3)$ \\
\hline Required respiratory support, \% (n) & $13.2(12)$ & $\mathrm{N} / \mathrm{A}$ & $48.0(12)$ \\
\hline Temperature, average ${ }^{\circ} \mathrm{F}$ (SEM) & $100.0(0.4)$ & $99.9(0.3)$ & $100.0(0.5)$ \\
\hline
\end{tabular}

Abbreviations: COVID-19 = coronavirus disease 2019; ICU = intensive care unit; N/A = not available; UNK = unknown.

COVID-19-associated symptoms and outcomes for each case are listed. All cases include all patients in the data set (second from the left column). These cases were then separated by hospitalization status (nonhospitalized [second from the right column] and hospitalized [right column]). There were significant differences between nonhospitalized and hospitalized patients by the $\chi^{2}$ or Mann-Whitney $U$ test with regard to dyspnea, headache, neurologic symptoms, and nausea/vomiting. Outcome metrics were not analyzed because most are not relevant to nonhospitalized patients, e.g., ICU admission or oxygen requirement. Values are expressed as either percentages with $\mathrm{n}$ in parenthesis or averages with SEM in parenthesis. The number of missing values for nonhospitalized patients was 51 for symptom duration, 53 for diagnosis duration, and 62 for temperature; and for hospitalized patients was 22 for symptom duration, 19 for diagnosis duration, and 7 for temperature.

a Significance of $p<0.05$. 


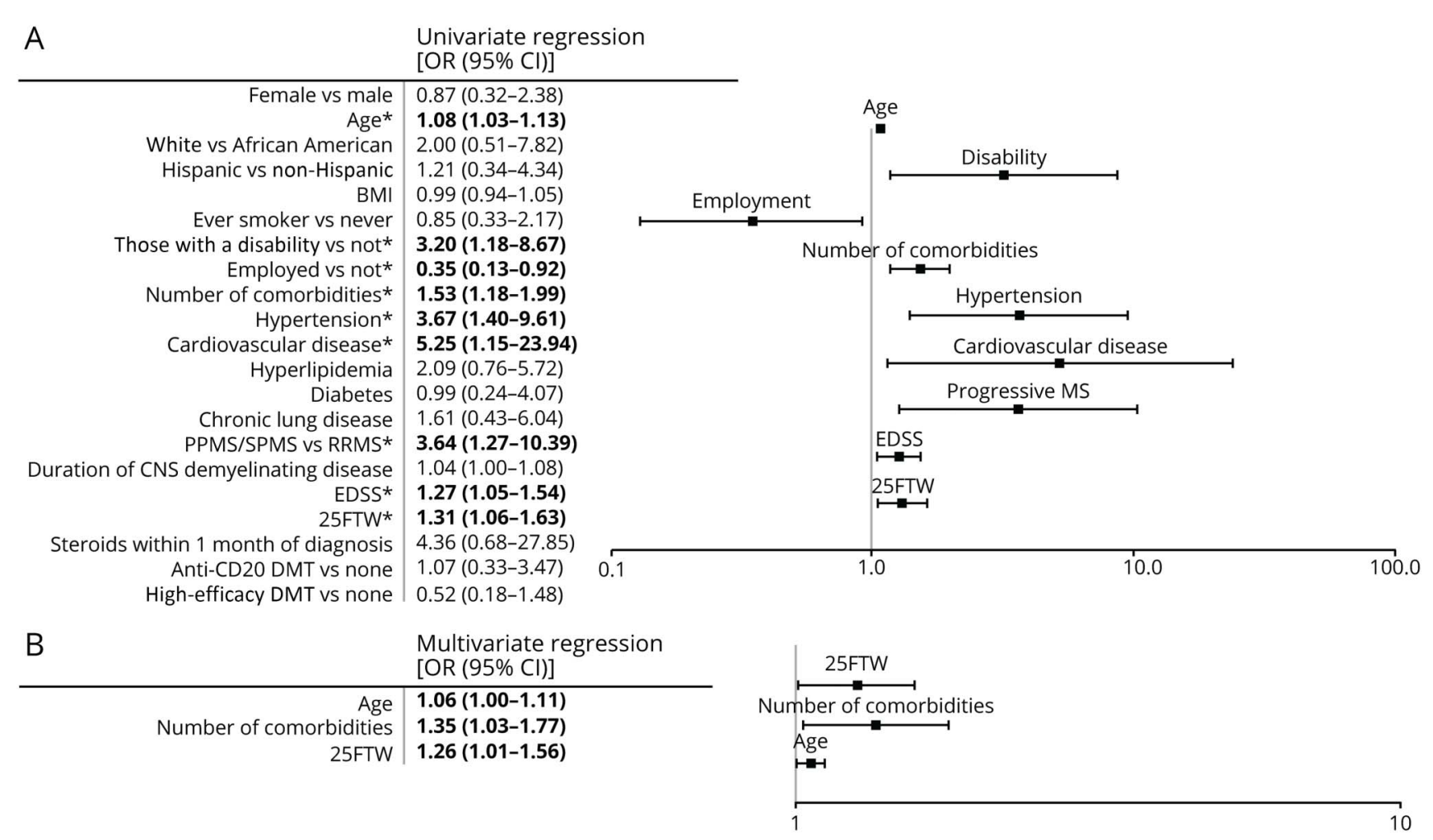

Univariate and multivariate logistic regression analyses were performed on patient characteristics before COVID-19 infection based on risk for hospitalization ( $n=66$ for nonhospitalized and $n=25$ for hospitalized). (A) Univariate logistic regression was performed on patient characteristics. ORs with $95 \%$ Cls are listed in the table to the left. Significant correlations are indicated by bold font and *. Age, disability, employment, number of non-neuroimmunologic comorbidities, hypertension, cardiovascular disease, progressive MS, and higher disability scores (EDSS and 25FTW) were predictive of hospitalization, whereas part-time or full-time employment predicted nonhospitalization. (B) Stepwise multivariate logistic regression utilizing Akaike information criteria was performed using significant hospitalization risk factors in univariate analysis. Only significant independent risk factors for hospitalization are listed in the table (left) and figure (right). Older age, a higher number of non-neuroimmunologic comorbidities, and a longer 25FTW were found to be independent risk factors for hospitalization. 25FTW = Timed 25-Foot Walk; BMI = body mass index; COVID-19 = coronavirus disease 2019; DMT = disease-modifying therapy; EDSS = Expanded Disability Status Scale; PPMS = primary progressive MS; RRMS = relapsing-remitting MS; SPMS = secondary progressive MS.

7.9 vs 6.2 seconds, $p<0.05)$ were more likely to require hospitalization. There was no significant difference in hospitalization rates among DMTs or when comparing DMT vs no DMT.

In terms of presenting COVID-19 symptoms, only dyspnea ( $40.0 \%$ vs $24.2 \%, p<0.05$ ), nausea/vomiting (32.0\% vs $7.6 \%, p$ $<0.01)$, and neurologic symptoms (56.0\% vs $13.6 \%, p<$ 0.0001 ) were more frequently reported by those hospitalized. Neurologic symptoms reported were vision change (1 patient), gait instability (3 patients), weakness (10 patients), speech difficulties (1 patient), dysphagia ( 1 patient), sensory symptoms (6 patients), urinary retention ( 1 patient), lethargy ( 1 patient), and hallucinations ( 1 patient). Only 2 of these (sensory symptoms and urinary retention) were associated with MS relapse per physician report. By contrast, headache (34.9\% vs $8.0 \%, p<0.01)$ was associated with nonhospitalization.

Univariate logistic regression analysis of clinical characteristics demonstrated a significant correlation of cardiovascular disease (OR 5.25, 95\% CI 1.15-23.94), hypertension (OR 3.67, 95\% CI 1.40-9.61), progressive MS (OR 3.64, 95\% CI 1.27-10.39), increased baseline disability (OR 3.20, 95\% CI 1.18-8.67), higher number of non-neuroimmunologic comorbidities (OR 1.53, 95\% CI 1.18-1.99), longer 25FTW (OR 1.31, 95\% CI 1.06-1.63), higher EDSS (OR 1.27, 95\% CI 1.05-1.54), and older age (OR 1.08, 95\% CI 1.03-1.13) with an increased risk of hospitalization. However, employment (OR 0.35, 95\% CI 0.13-0.92) correlated with a decreased risk (Figure 1). When these factors were analyzed with a stepwise multivariate logistic regression, only number of non-neuroimmunologic comorbidities (OR 1.35, 95\% CI 1.03-1.77), 25FTW (OR 1.26, 95\% CI 1.01-1.56), and older age (OR 1.06, 95\% CI 1.00-1.11) were independent risk factors for hospitalization (Figure 2).

Univariate analysis of symptoms demonstrated a similar pattern as above with headache (OR $0.16,95 \%$ CI 0.04-0.75) associated with a decreased hospitalization risk, whereas neurologic symptoms (OR 8.06, 95\% CI 2.80-23.20), nausea/vomiting (OR 5.74, 95\% CI 1.66-19.84), and dyspnea (OR 2.88, 95\% CI 1.10-7.58) were associated with an increased risk of hospitalization. However, only headache (OR 0.08, 95\% CI 0.01-0.57), neurologic symptoms (OR 8.57, 95\% CI 2.49-29.48), and nausea/vomiting (OR 7.24, 95\% CI 1.66-31.54) were independent risk factors when analyzed in a stepwise multivariate regression. 
Figure 2 Univariate and Multivariate Regressions of Presenting Symptoms for the Risk of Hospitalization

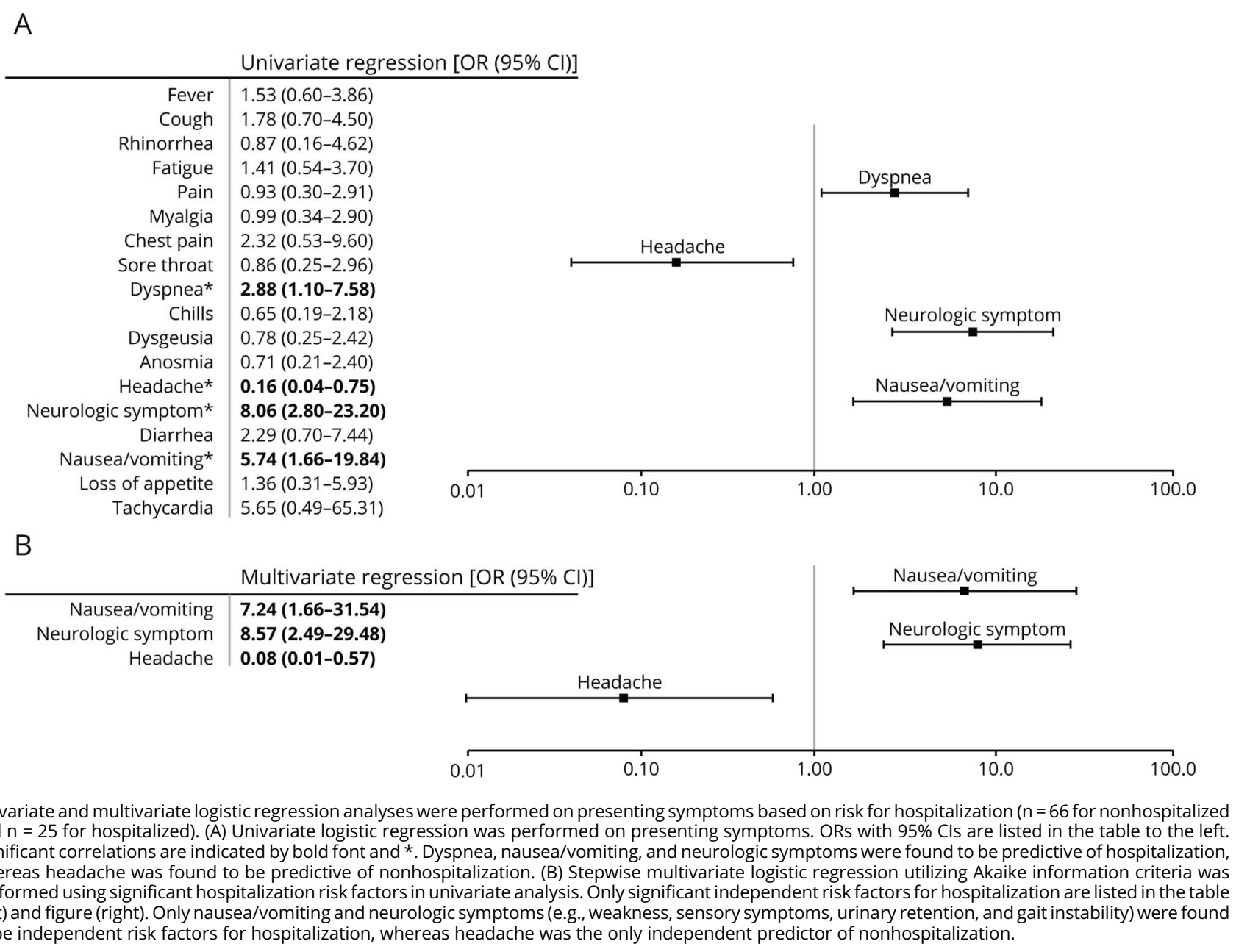

Laboratory values were only available for a limited number of patients preceding, during, and after COVID-19 infection, with values listed in eTable 1 (links.lww.com/NXI/A513) and significant values included in Figure 3. We found a significant association between absolute lymphocyte count (ALC) and risk of hospitalization. In addition, ALC was significantly lower in hospitalized patients compared with nonhospitalized COVID-19 patients (Figure 3). No significant differences in vitamin $\mathrm{D}$ levels were noted by either time or hospitalization status, which is confounded by the small number of values available for during and after infection. Hemoglobin was found to be significantly lower at all time points in the hospitalized group, and albumin decreased during and after COVID-19 infection in the hospitalized group. Aspartate transaminase was higher during COVID-19 infection in both groups.

\section{Effect of DMT on COVID-19 Severity and Duration}

No effect of DMT on hospitalization risk was found irrespective of the comparison made, that is, presence of DMT vs absence of DMT, anti-CD20 medications vs no DMT, and high- vs mid- vs low-efficacy DMT. However, all 12 patients with respiratory failure requiring supplemental oxygen, noninvasive positive pressure ventilation, or intubation were on a DMT $(p<0.05)$ with half of them on an anti-CD20 agent. Although the reason for DMT dose changes was not specified, our cohort does include 14 patients with delayed or extended interval dosing of their DMT during COVID-19 infection. Dimethyl fumarate was transiently held during infection for 3 patients with one experiencing a pseudorelapse while held and another requiring hospitalization for COVID-19 infection. Eight patients on natalizumab had a delayed dose or transitioned to extended interval dosing (every 6 weeks) with none experiencing a relapse or pseudorelapse and none requiring hospitalization for COVID-19 infection. Doses were delayed or missed for 3 patients on ocrelizumab, but none experienced relapse or pseudorelapse and only 1 required hospitalization for COVID-19 infection. Analysis of these dosing changes was not statistically possible.

Of interest, although there was no difference with regard to symptom duration based on relative DMT efficacy, COVID-19 PCR positivity duration was significantly shorter for patients on high-efficacy DMTs compared with no/other DMT (Figure 4). Of the patients with COVID-19 antibody testing, 13 were 
Figure 3 Significant Laboratory Values Before/During/After COVID-19 Infection

\begin{tabular}{|c|c|c|c|c|c|}
\hline \multicolumn{2}{|l|}{ A } & \multicolumn{2}{|c|}{ Nonhospitalized [average (SEM)] } & \multicolumn{2}{|c|}{ Hospitalized [average (SEM)] } \\
\hline \multirow{3}{*}{$\begin{array}{l}\text { Absolute } \\
\text { lymphocyte } \\
\text { count }\end{array}$} & Before & $1.69(0.12)$ & $\mathrm{n}=48$ & $1.45(0.18)$ & $\mathrm{n}=19$ \\
\hline & During & $2.13(0.36)^{*}$ & $\mathrm{n}=15$ & $0.85(0.10)^{*}$ & $\mathrm{n}=16$ \\
\hline & After & $1.92(0.14)$ & $n=30$ & $1.78(0.32)$ & $\mathrm{n}=10$ \\
\hline \multirow{3}{*}{ Hemoglobin } & Before & $13.23(0.21)$ & $\mathrm{n}=49$ & $12.71(0.42)$ & $\mathrm{n}=19$ \\
\hline & During & $13.05(0.33)$ & $\mathrm{n}=15$ & $12.36(0.46)$ & $\mathrm{n}=17$ \\
\hline & After & $13.73(0.23)$ & $\mathrm{n}=31$ & $12.03(0.57)$ & $\mathrm{n}=11$ \\
\hline \multirow{3}{*}{$\begin{array}{c}\text { Aspartate } \\
\text { transaminase }\end{array}$} & Before & $19.93(1.16)$ & $\mathrm{n}=47$ & $16.11(1.68)$ & $\mathrm{n}=19$ \\
\hline & During & $22.50(2.98)$ & $n=10$ & $31.25(5.62)$ & $\mathrm{n}=16$ \\
\hline & After & $17.95(1.32)$ & $\mathrm{n}=20$ & $19.50(2.40)$ & $n=4$ \\
\hline \multirow{3}{*}{ Albumin } & Before & $4.34(0.09)$ & $\mathrm{n}=37$ & $4.39(0.20)$ & $\mathrm{n}=17$ \\
\hline & During & $4.17(0.10)$ & $\mathrm{n}=9$ & $3.71(0.18)$ & $\mathrm{n}=12$ \\
\hline & After & $4.38(0.06)$ & $n=15$ & $3.80(0.12)$ & $n=3$ \\
\hline
\end{tabular}

$\mathrm{B}$

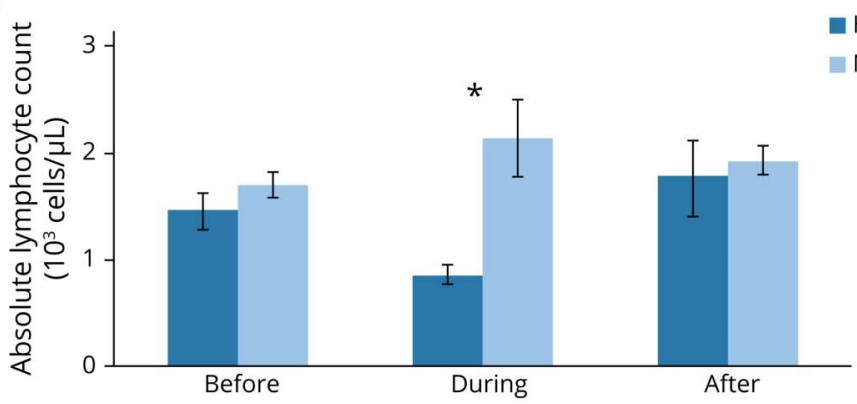

Laboratory tests were obtained before, during, and after COVID-19 infection. (A) Laboratory values before, during, and after COVID-19 infection grouped by hospitalization status (nonhospitalized [left] and hospitalized [right]) were analyzed for significant effects of time and/or hospitalization. Values are listed as averages with SEM in parenthesis, and the rightward column indicating the number of values included in that average. Values represent unique contributions from 49 nonhospitalized patients and 19 hospitalized patients. Significance was determined by two-way analysis of variance, with significance between each group determined by Bonferroni post hoc analysis. Significant effect of time was found for aspartate transaminase. Significant effect of hospitalization status was found for absolute lymphocyte count, hemoglobin, and albumin. There was a significant interaction between time and hospitalization status for absolute lymphocyte count with post hoc analysis indicating significance during hospitalization. Post hoc analysis did not indicate a significant individual interaction for hemoglobin, aspartate transaminase, or albumin. Significant $(p<0.05)$ effect of subgroups in post hoc analysis is indicated by *. (B) Absolute lymphocyte count was lower at all time points in the hospitalized group. There were also unique trends in each group, with nonhospitalized patients having a peak during acute infection, whereas hospitalized patients demonstrated a nadir during acute infection. Error bars represent SEM. Significance through Bonferroni post hoc analysis is indicated by *. COVID-19 = coronavirus disease 2019.

negative (6 of whom were not PCR-confirmed cases), and 18 were positive, for a sensitivity of 0.58 . The average time between the earliest symptom or positive PCR and antibody testing was 83.4 days. COVID-19 antibody positivity was not significantly affected either by individual DMT or by efficacy-grouped DMT, suggesting that the DMT type does not affect the ability to form antibodies to COVID-19 (eFigure 1, links.lww.com/NXI/A513).

\section{Trends in Patients With More Severe COVID-19}

Although there were insufficient numbers to analyze risk factors associated with more severe COVID-19 infection features, such as ICU admission and fatality, some trends can be appreciated in this cohort. The 5 patients admitted to an ICU were older (average age 56.4 years) with primary or secondary progressive MS (80\%) and were more likely to be on disability $(60 \%)$. This group was also notable for higher disability scores (average EDSS 7.0 and average 25FTW 7.3 seconds), current/prior smokers (80\%), and presenting symptoms of dyspnea $(60 \%)$ and/or tachycardia $(20 \%)$.
Of the 4 fatalities in our cohort, 3 were hospitalized and 1 required ICU-level care. These patients were older (average age 72.5 years), White, and non-Hispanic. Three were male, 3 had primary or secondary progressive MS, and all were unemployed (either on disability or retired). One patient was not on DMT, 1 on cladribine and glatiramer acetate, 1 on monthly IV methylprednisolone, and 1 on tacrolimus (for MS and liver transplant).

\section{Discussion}

In this article, we demonstrate a mostly mild to moderate severity COVID-19 disease course in our CNS demyelinating disease cohort. This is similar to other case reports/series and small cohort studies of patients with MS from around the world demonstrating a norm of mildmoderate COVID-19 disease severity. ${ }^{9-12,14,15}$ Our findings show that clinical characteristics and laboratory abnormalities associated with increased COVID-19 severity were similar to those observed in the general population but also 
A. Symptom duration by DMT efficacy

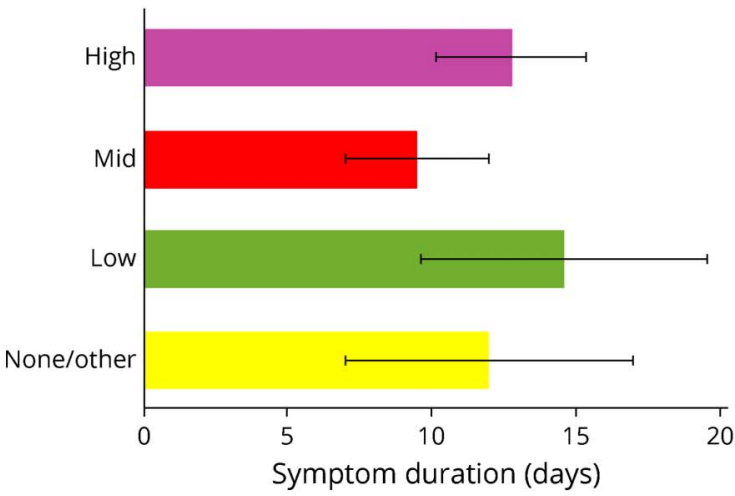

B. PCR positivity duration by DMT efficacy

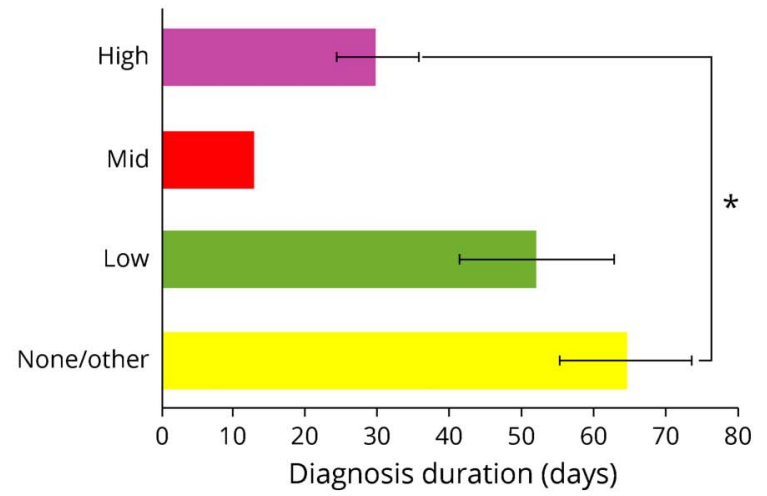

Duration of COVID-19 symptoms and duration of COVID-19 PCR positivity were grouped by DMT efficacy (none/other, low, mid, and high). (A) Symptom duration in days is indicated on the $x$ axis, and DMTs grouped by efficacy are on the $y$ axis. There were no significant differences in symptom duration based on DMT efficacy with one-way analysis of variance. A number of patients contributing to each group for symptom duration were 2 none/other, 5 low efficacy, 2 mid efficacy, and 10 high efficacy. (B) Duration of PCR positivity in days is indicated on the $x$ axis, and DMTs grouped by efficacy are on the y axis. There was a significant association of DMT efficacy with post hoc analysis (Bonferroni) demonstrating a significant difference between high-efficacy DMT vs none/other DMT with regard to duration of PCR positivity. A number of patients contributing to each group for PCR positivity duration were 4 none/other, 5 low efficacy, 1 mid efficacy, and 9 high efficacy. Error bars indicated SEM and are not shown in samples where $\mathrm{n}=1$. Significance $(p<0.05)$ is indicated by *. COVID-19= coronavirus disease 2019; DMT = disease-modifying therapy.

that there were a unique set of symptoms associated with hospitalization risk in our cohort (i.e., neurologic symptoms, nausea/vomiting, and dyspnea).,3

Although our case fatality rate of $4.4 \%$ is higher than some MS cohorts, it is similar to the case fatality rate within the northeastern US and other major US cities. ${ }^{13,16,17}$ All 4 fatalities seen in our cohort were older patients with disability with multiple comorbidities. Their severe COVID-19 course may have been predictable by these known risk factors. ${ }^{13,18,19}$ These patients were not on high-efficacy DMTs, and the most likely contributor to severe COVID-19 was more severe baseline neurologic disability.

Our data support continuing DMTs during the COVID-19 pandemic. There were no correlations or associations of DMT with COVID-19 hospitalization, no difference in antibody formation by the DMT type, and decreased COVID-19 duration with high-efficacy DMTs. There was also no association between progressive MS and either anti-CD20 agent or high-efficacy DMT use to suggest that these factors are linked. Despite prior report of increased severity COVID-19 on anti-CD20 agents, we were unable to confirm this in our cohort. $^{20}$ Several case reports have demonstrated a mild COVID-19 course with rituximab, ${ }^{21-25}$ ocrelizumab, ${ }^{26-28}$ teriflunomide, $^{29}$ and natalizumab. ${ }^{30,31}$ Worsening COVID-19 symptoms after withdrawal of fingolimod have been reported, ${ }^{32-34}$ which may be a product of rebound inflammation associated specifically with fingolimod. ${ }^{35,36}$ All patients requiring respiratory support were on a DMT in our study, but we require further data to examine whether classes or individual DMTs are associated with different COVID-19 outcomes.
Clinical characteristics associated with COVID-19 severity in our cohort, such as older age and number of comorbidities, are similar to those observed for the general population. ${ }^{2,3}$ Higher rates of COVID-19 hospitalization with increased disability have been observed in other MS cohorts as well. ${ }^{9,13}$ The association of headache with a decreased likelihood of COVID-19 hospitalization suggests, for unclear reasons, this symptom portends a milder course. ${ }^{9}$

For our patient cohort, we found a significantly higher proportion of neurologic symptoms in the hospitalized group. Neurologic symptoms reported include weakness, paresthesia, gait imbalance, urinary retention, dysarthria, dysphagia, and blurry vision and were nearly all considered symptoms of pseudorelapse. ${ }^{9}$ By contrast, we found only 2 MS clinical relapses during or after COVID-19 infection.

Several laboratory abnormalities have been associated with COVID-19 infection, most notably decreased ALC. ${ }^{2}$ We found that hospitalized patients had a significantly decreased ALC compared with nonhospitalized patients during symptomatic COVID-19 infection. The significance of this finding was unaffected by removal of patients on the lymphocyte-sequestering agent fingolimod. Decreased ALC during acute illness likely reflects increased COVID-19 severity and lymphocyte recruitment to target organs. ${ }^{2}$

Our study is not without limitations. MS providers entered data electronically based on EMRs and only included patients with CNS demyelinating disease with suspected/confirmed COVID-19, which could have created a sampling bias. As a result of differences in data collection, multiple imputations were used for variables such as BMI, EDSS, and 25FTW. 
Although available, data for some factors (e.g., COVID19-directed treatments) had limited sample size to be analyzed sufficiently. Treatments received for COVID-19 could affect the outcome, and we are compiling these data for future analysis. There has been insufficient follow-up to assess evidence of progression or accumulated disability in this cohort after COVID-19 infection. Finally, we only analyzed patients who developed COVID-19, so we are unable to assess factors that influence susceptibility to COVID-19 infection.

We present these findings as a pilot analysis while data collection remains ongoing. The overall mild course of COVID-19 observed in most patients with CNS demyelinating disease to date is reassuring and suggests that MS and its treatment do not increase the risk for severe COVID-19 infection beyond the known risk factors identified in the general population (i.e., older age, disability, and increased number of non-neuroimmunologic comorbidities). However, there may be subgroups of patients who would benefit from an alternate course of treatment, such as extended interval dosing of some DMTs, which we have yet to delineate. As we continue to collect data, we intend to better identify the effect of individual DMTs and to observe the effects of COVID-19 infection on CNS demyelinating disease progression and disability over time. We are actively collecting follow-up data on patients within this cohort to be included in future studies. We also hope to demonstrate the influence of DMTs on vaccine efficacy as they become widely available. As COVID-19 continues to surge, care of patients with CNS demyelinating diseases such as MS must evolve based on data as it becomes available, and we hope to continue to add to this with future studies.

\section{Study Funding}

The authors report no targeted funding.

\section{Disclosure}

K.M. Money, A. Mahatoo, S. Samaan, P. Anand, U. Baber, and $\mathrm{M}$. Bailey report no disclosures relevant to the manuscript. R. Bakshi has received consulting fees from EMD Serono and research funding from BMS/Celgene and EMD Serono. A. Bouley and A. Bower report no disclosures relevant to the manuscript. J. Cahill has research funding from Roche. E. Lathi reports no disclosures relevant to the manuscript. E.E. Longbrake has consulted for Genentech, Genzyme, Alexion, Biogen, EMD, and Serono. M. McAdams, S. Napoli, and P. Raibagkar report no disclosures relevant to the manuscript. J.A. Sloane has grant funding from the National MS Society, Biogen, and Genentech and consulted for Biogen, Genentech, Teva, Banner, Sanofi, and Celgene. Go to Neurology.org/NN for full disclosures.

\section{Publication History}

Received by Neurology: Neuroimmunology \& Neuroinflammation February 13, 2021. Accepted in final form June 1, 2021.

\section{Appendix Authors}

\begin{tabular}{lll}
\hline Name & Location & Contribution \\
\hline $\begin{array}{l}\text { Kelli M. } \\
\text { Money, MD, } \\
\text { PhD }\end{array}$ & $\begin{array}{l}\text { Beth Israel Deaconess } \\
\text { Medical Center, Boston, MA }\end{array}$ & $\begin{array}{l}\text { Drafting/revision of the } \\
\text { manuscript for content, } \\
\text { including medical writing for } \\
\text { content; and analysis or } \\
\text { interpretation of data }\end{array}$ \\
\hline $\begin{array}{l}\text { Ashmanie } \\
\text { Mahatoo, }\end{array}$ & Beth Israel Deaconess & $\begin{array}{l}\text { Drafting/revision of the } \\
\text { manuscript for content, } \\
\text { including medical writing for } \\
\text { content }\end{array}$ \\
\hline $\begin{array}{l}\text { Soleil } \\
\text { Samaan }\end{array}$ & Beth Israel Deaconess & $\begin{array}{l}\text { Drafting/revision of the } \\
\text { manuscript for content, } \\
\text { including medical writing for } \\
\text { content; and major role in } \\
\text { the acquisition of data }\end{array}$ \\
\hline
\end{tabular}

Pria Anand, Boston University School of Drafting/revision of the MD Medicine, Boston, MA manuscript for content, including medical writing for content; and major role in the acquisition of data

\begin{tabular}{lll}
\hline Ursela & Beth Israel Deaconess & $\begin{array}{l}\text { Drafting/revision of the } \\
\text { Baber, MD }\end{array}$ \\
Medical Center, Boston, MA & $\begin{array}{l}\text { mancript for content, } \\
\text { including medical writing for } \\
\text { content; and major role in } \\
\text { the acquisition of data }\end{array}$
\end{tabular}

Mary St. Francis Hospital, Hartford, Drafting/revision of the Bailey, MD CT manuscript for content, including medical writing for content; and major role in the acquisition of data

\begin{tabular}{lll}
\hline Rohit & Brigham and Women's & $\begin{array}{l}\text { Drafting/revision of the } \\
\text { makshi, MD }\end{array}$ \\
Hospital, Boston, MA & $\begin{array}{l}\text { including medical writing for } \\
\text { content; and major role in } \\
\text { the acquisition of data }\end{array}$
\end{tabular}

\begin{tabular}{lll}
\hline $\begin{array}{l}\text { Andrew } \\
\text { Bouley, MD }\end{array}$ & $\begin{array}{l}\text { Elliot Lewis Center, Wellesley, } \\
\text { MA }\end{array}$ & $\begin{array}{l}\text { Major role in the acquisition } \\
\text { of data }\end{array}$ \\
\hline $\begin{array}{l}\text { Aaron } \\
\text { Bower, MD }\end{array}$ & $\begin{array}{l}\text { Yale School of Medicine, New } \\
\text { Haven, CT }\end{array}$ & $\begin{array}{l}\text { Drafting/revision of the } \\
\text { manuscript for content, } \\
\text { including medical writing for } \\
\text { content; and major role in } \\
\text { the acquisition of data }\end{array}$ \\
\hline $\begin{array}{l}\text { Jonathan } \\
\text { Cahill, MD }\end{array}$ & $\begin{array}{l}\text { Rhode Island Hospital, } \\
\text { Providence, RI }\end{array}$ & $\begin{array}{l}\text { Drafting/revision of the } \\
\text { manuscript for content, } \\
\text { including medical writing for } \\
\text { content; and major role in } \\
\text { the acquisition of data }\end{array}$ \\
& &
\end{tabular}

\begin{tabular}{|c|c|c|}
\hline $\begin{array}{l}\text { Maria } \\
\text { Houtchens, } \\
\text { MD }\end{array}$ & $\begin{array}{l}\text { Brigham and Women's } \\
\text { Hospital, Boston, MA }\end{array}$ & $\begin{array}{l}\text { Drafting/revision of the } \\
\text { manuscript for content, } \\
\text { including medical writing for } \\
\text { content; and major role in } \\
\text { the acquisition of data }\end{array}$ \\
\hline $\begin{array}{l}\text { Joshua } \\
\text { Katz, MD }\end{array}$ & $\begin{array}{l}\text { Elliot Lewis Center, Wellesley, } \\
\text { MA }\end{array}$ & $\begin{array}{l}\text { Drafting/revision of the } \\
\text { manuscript for content, } \\
\text { including medical writing for } \\
\text { content; and major role in } \\
\text { the acquisition of } \\
\text { data }\end{array}$ \\
\hline $\begin{array}{l}\text { Ellen Lathi, } \\
\text { MD }\end{array}$ & $\begin{array}{l}\text { Elliot Lewis Center, Wellesley, } \\
\text { MA }\end{array}$ & $\begin{array}{l}\text { Drafting/revision of the } \\
\text { manuscript for content, } \\
\text { including medical writing for } \\
\text { content; and major role in } \\
\text { the acquisition of } \\
\text { data }\end{array}$ \\
\hline
\end{tabular}


Appendix (continued)

\begin{tabular}{lll}
\hline Name & Location & Contribution \\
\hline $\begin{array}{l}\text { Elle Levit, } \\
\text { MD }\end{array}$ & $\begin{array}{l}\text { Yale School of Medicine, New } \\
\text { Haven, CT }\end{array}$ & $\begin{array}{l}\text { Drafting/revision of the } \\
\text { manuscript for content, } \\
\text { including medical writing for } \\
\text { content; and major role in } \\
\text { the acquisition of data }\end{array}$ \\
\hline $\begin{array}{l}\text { Erin E. } \\
\text { Longbrake, } \\
\text { MD }\end{array}$ & Yale School of Medicine, New $C T$ & $\begin{array}{l}\text { Drafting/revision of the } \\
\text { manuscript for content, } \\
\text { including medical writing for } \\
\text { content; and major role in } \\
\text { the acquisition of data }\end{array}$
\end{tabular}

\begin{tabular}{lll}
\hline Matthew & Lahey Hospital, Burlington, & Drafting/revision of the \\
McAdams, & MA & $\begin{array}{l}\text { manuscript for content, } \\
\text { including medical writing for } \\
\text { MD }\end{array}$ \\
& & content; and major role in \\
& the acquision of data
\end{tabular}

\begin{tabular}{ll}
\hline Salvatore & Neurology Center of New \\
Napoli, MD & England, Foxboro, MA
\end{tabular}

the acquisition of data

Drafting/revision of the manuscript for content, including medical writing for content; and major role in the acquisition of data

\begin{tabular}{lll}
\hline Pooja & Formerly Lahey Hospital, & $\begin{array}{l}\text { Drafting/revision of the } \\
\text { Raibagkar, } \\
\text { MD }\end{array}$ \\
$\begin{array}{l}\text { Burlington, MA; now at } \\
\text { Concord Hospital, Concord, }\end{array}$ & $\begin{array}{l}\text { including medical writing for } \\
\text { in }\end{array}$ & $\begin{array}{l}\text { content; and major role in } \\
\text { the acquisition of data }\end{array}$
\end{tabular}

Peter Wade, St. Francis Hospital, Hartford, Drafting/revision of the MD CT manuscript for content, including medical writing for content; and major role in the acquisition of data

\begin{tabular}{ll}
\hline Jacob A. & Beth Israel Deaconess \\
Sloane, MD, & Medical Center, Boston, MA \\
PhD &
\end{tabular}

Drafting/revision of the manuscript for content, including medical writing for content; major role in the acquisition of data; study concept or design; and analysis or interpretation of data

\section{References}

1. COVID-19 Map. Johns Hopkins Coronavirus Resource Center. Accessed January 13, 2021. https://coronavirus.jhu.edu/map.html.

2. Richardson $S$, Hirsch JS, Narasimhan M, et al. Presenting characteristics, comorbidities, and outcomes among 5700 patients hospitalized with COVID-19 in the New York City area. JAMA. 2020;323(20):2052-2059.

3. Wu Z, McGoogan JM. Characteristics of and important lessons from the coronavirus disease 2019 (COVID-19) outbreak in China: summary of a report of 72314 cases from the Chinese Center for Disease Control and Prevention. JAMA. 2020;323(13):1239.

4. Luna G, Alping P, Burman J, et al. Infection risks among patients with multiple sclerosis treated with fingolimod, natalizumab, rituximab, and injectable therapies. JAMA Neurol. 2020;77(2):184-191.

5. Winkelmann A, Loeleebermann M, Reisinger EC, Hartung H-P, Zettl UK. Diseasemodifying therapies and infectious risks in multiple sclerosis. Nat Rev Neurol. 2016; 12(4):217-233.

6. Vogel AC, Schmidt H, Loud S, McBurney R, Mateen FJ. Impact of the COVID-19 pandemic on the health care of $>1,000$ people living with multiple sclerosis: a crosssectional study. Mult Scler Relat Disord. 2020;46:102512.

7. Giovannoni G, Hawkes C, Lechner-Scott J, Levy M, Waubant E, Gold J. The COVID19 pandemic and the use of MS disease-modifying therapies. Mult Scler Relat Disord. 2020;39:102073.

8. The coronavirus and MS - updated global advice. MS Int Fed. 2020. Accessed November 23, 2020. msif.org/news/2020/02/10/the-coronavirus-and-ms-what-you-need-to-know/.
9. Parrotta E, Kister I, Charvet L, et al. COVID-19 outcomes in MS. Neurol Neuroimmunol Neuroinflammation. 2020;7(5):e835.

10. Chaudhry F, Bulka H, Rathnam AS, et al. COVID-19 in multiple sclerosis patients and risk factors for severe infection. J Neurol Sci. 2020;418:117147.

11. Sormani MP. An Italian programme for COVID-19 infection in multiple sclerosis. Lancet Neurol. 2020;19(6):481-482.

12. Crescenzo F, Marastoni D, Bovo C, Calabrese M. Frequency and severity of COVID19 in multiple sclerosis: a short single-site report from northern Italy. Mult Scler Relat Disord. 2020;44:102372.

13. Louapre C, Collongues N, Stankoff B, et al. Clinical characteristics and outcomes in patients with coronavirus disease 2019 and multiple sclerosis. JAMA Neurol. 2020; 77(9):1079-1088.

14. Mantero V, Abate L, Balgera R, Basilico P, Salmaggi A, Cordano C. Assessing the susceptibility to acute respiratory illness COVID-19-related in a cohort of multiple sclerosis patients. Mult Scler Relat Disord. 2020;46:102453.

15. Möhn N, Konen FF, Pul R, et al. Experience in multiple sclerosis patients with COVID-19 and disease-modifying therapies: a review of 873 published cases. J Clin Med. 2020;9(12):4067.

16. Suleyman G, Fadel RA, Malette KM, et al. Clinical characteristics and morbidity associated with coronavirus disease 2019 in a series of patients in metropolitan Detroit. JAMA Netw Open. 2020;3(6):e2012270.

17. Garibaldi BT, Fiksel J, Muschelli J, et al. Patient trajectories among persons hospitalized for COVID-19. Ann Intern Med. 2021;147(1):33-41.

18. Davies NG, Klepac P, Liu Y, et al. Age-dependent effects in the transmission and control of COVID-19 epidemics. Nat Med. 2020;26(8):1205-1211.

19. Iaccarino G, Grassi G, Borghi C, et al. Age and multimorbidity predict death among COVID-19 patients: results of the SARS-RAS study of the Italian Society of Hypertension. Hypertension. 2020;76(2):366-372.

20. Sormani MP, De Rossi N, Schiavetti I, et al. Disease modifying therapies and Covid-19 severity in multiple sclerosis. Ann Neurol. 2021;89(4):780-789.

21. Montero-Escribano P, Matías-Guiu J, Gómez-Iglesias P, Porta-Etessam J, Pytel V, Matias-Guiu JA. Anti-CD20 and COVID-19 in multiple sclerosis and related disorders: a case series of 60 patients from Madrid, Spain. Mult Scler Relat Disord. 2020;42: 102185.

22. Wurm H, Attfield K, Iversen AK, Gold R, Fugger L, Haghikia A. Recovery from COVID-19 in a B-cell-depleted multiple sclerosis patient. Mult Scler. 2020;26(10): 1261-1264.

23. Devogelaere J, D'hooghe MB, Vanderhauwaert F, D'haeseleer M. Coronavirus disease 2019: favorable outcome in an immunosuppressed patient with multiple sclerosis. Neurol Sci. 2020;41(8):1981-1983.

24. Creed MA, Ballesteros E, Jr LJG, Imitola J. Mild COVID-19 infection despite chronic $B$ cell depletion in a patient with aquaporin-4-positive neuromyelitis optica spectrum disorder. Mult Scler Relat Disord. 2020;44:102199.

25. Hughes R, Pedotti R, Koendgen H. COVID-19 in persons with multiple sclerosis treated with ocrelizumab - a pharmacovigilance case series. Mult Scler Relat Disord. 2020;42:102192.

26. Novi G, Mikulska M, Briano F, et al. COVID-19 in a MS patient treated with ocrelizumab: does immunosuppression have a protective role? Mult Scler Relat Disord. 2020;42:102120.

27. Ghajarzadeh M, Mirmosayyeb O, Barzegar M, et al. Favorable outcome after COVID19 infection in a multiple sclerosis patient initiated on ocrelizumab during the pandemic. Mult Scler Relat Disord. 2020;43:102222.

28. Thornton JR, Harel A. Negative SARS-CoV-2 antibody testing following COVID-19 infection in Two MS patients treated with ocrelizumab. Mult Scler Relat Disord. 2020; 44(2):102341.

29. Maghzi AH, Houtchens MK, Preziosa P, et al. COVID-19 in teriflunomide-treated patients with multiple sclerosis. J Neurol. 2020;267(10):2790-2796.

30. Aguirre C, Meca-Lallana V, Barrios-Blandino A, del Río B, Vivancos J. Covid-19 in a patient with multiple sclerosis treated with natalizumab: may the blockade of integrins have a protective role? Mult Scler Relat Disord. 2020;44:102250.

31. Borriello G, Ianniello A. COVID-19 occurring during natalizumab treatment: a case report in a patient with extended interval dosing approach. Mult Scler Relat Disord. 2020;41:102165.

32. Gomez-Mayordomo V, Montero-Escribano P, Matías-Guiu JA, González-García N, Porta-Etessam J, Matías-Guiu J. Clinical exacerbation of SARS-CoV2 infection after fingolimod withdrawal. J Med Virol. 2021;93(1):546-549.

33. Valencia-Sanchez C, Wingerchuk DM. A fine balance: immunosuppression and immunotherapy in a patient with multiple sclerosis and COVID-19. Mult Scler Relat Disord. 2020;42:102182.

34. Foerch C, Friedauer L, Bauer B, Wolf T, Adam EH. Severe COVID-19 infection in a patient with multiple sclerosis treated with fingolimod. Mult Scler Relat Disord. 2020; 42:102180.

35. Havla JB, Pellkofer HL, Meinl I, Gerdes LA, Hohlfeld R, Kümpfel T. Rebound of disease activity after withdrawal of fingolimod (FTY720) treatment. Arch Neurol. 2012;69(2):262-264.

36. Barry B, Erwin AA, Stevens J, Tornatore C. Fingolimod rebound: a review of the clinical experience and management considerations. Neurol Ther. 2019;8(2):241-250. 


\title{
Neurology \\ Neuroimmunology \& Neuroinflammation
}

\author{
A New England COVID-19 Registry of Patients With CNS Demyelinating Disease: A \\ Pilot Analysis \\ Kelli M. Money, Ashmanie Mahatoo, Soleil Samaan, et al. \\ Neurol Neuroimmunol Neuroinflamm 2021;8; \\ DOI 10.1212/NXI.0000000000001046
}

This information is current as of August 2, 2021

\section{Updated Information \& \\ Services}

References

Citations

Subspecialty Collections

Permissions \& Licensing

Reprints including high resolution figures, can be found at:

http://nn.neurology.org/content/8/5/e1046.full.html

This article cites 34 articles, 0 of which you can access for free at: http://nn.neurology.org/content/8/5/e1046.full.html\#\#ref-list-1

This article has been cited by 2 HighWire-hosted articles:

http://nn.neurology.org/content/8/5/e1046.full.html\#\#otherarticles

This article, along with others on similar topics, appears in the following collection(s):

All Demyelinating disease (CNS)

http://nn.neurology.org//cgi/collection/all_demyelinating_disease_cns

Cohort studies

http://nn.neurology.org//cgi/collection/cohort_studies

COVID-19

http://nn.neurology.org//cgi/collection/covid_19

Information about reproducing this article in parts (figures,tables) or in its entirety can be found online at:

http://nn.neurology.org/misc/about.xhtml\#permissions

Information about ordering reprints can be found online:

http://nn.neurology.org/misc/addir.xhtml\#reprintsus

Neurol Neuroimmunol Neuroinflamm is an official journal of the American Academy of Neurology.

Published since April 2014, it is an open-access, online-only, continuous publication journal. Copyright

Copyright $\odot 2021$ The Author(s). Published by Wolters Kluwer Health, Inc. on behalf of the American

Academy of Neurology.. All rights reserved. Online ISSN: 2332-7812.

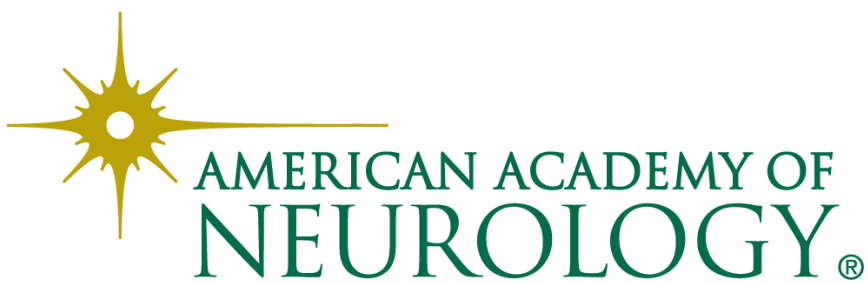

